\title{
Feasibility Study on Shared Employees Based on Blockchain Technology
}

\author{
Xin Peng* \\ Department of Economics and Management, Beijing City University, \\ Muyan Road, Yang Town, Shunyi District, Beijing, China \\ xinpeng@bcu.edu.cn \\ Received 1 December 2021; Revised 2 January 2022; Accepted 2 February 2022
}

\begin{abstract}
Blockchain is a distributed ledger formed by sequentially adding consensual blocks linked by cryptography. Which create a reliable cooperation mechanism for shared employees because it can be traceable and cannot be forged. The shared employees was born in 2020 as a temporary measure to address the imbalance in personnel allocation among certain industries amid the spread of COVID-19. With the help of blockchain technology, shared employees can achieve rapid development in the establishment of trust mechanism, traceability of employees' background, and the establishment of credit evaluation system. The new economic model of shared employees supported by blockchain technology may bring benefits to the transformation of employment mode, the upgrading of industrial structure and even the economic integration of urban and rural areas in China.
\end{abstract}

Keywords: blockchain, shared employees, smart contract, credit evaluation system

\section{Introduction}

Shared economy is a new form that relying on information technology innovation and marketization. It can make the mechanism of resource allocation more convenient, flexible and efficient, can give full play to the potential of various resource factors. Its birth is an inevitable outcome of the development of science and technology. From the end of 2019 to 2021, the COVID-19 outbreak, which has brought great challenges to labor and employment in China and even the world. During the epidemic period, people have to study and work at home, resulting in a sharp decline in the income of offline catering industries such as education, training and hotel accommodation. At the same time, some other industries saw a surge in orders, such as fresh e-commerce and logistics distribution enterprises. As a result, these industries are facing the problem of manpower shortage. This provides the conditions for the birth of "shared employees". In February 2020, Hema Fresh Supermarket of Alibaba Group took the first step of "shared employees", they reached cooperation with Xibei, Yunhaiyao and Youth Restaurant (Beijing), and invited employees of these enterprises to work in Hema during the epidemic period. Thus opened the curtain of "shared employees". In 2021, the epidemic recurred, and Hema's shared employee platform went online the second time [1].

Blockchain is a distributed infrastructure and computing paradigm formed by the highly integrated cryptographic algorithm, consensus mechanism, point-to-point communication protocol, distributed storage and other core technology [2]. Its four features guarantee the safe and efficient operation of the shared employees economic model: (1) Immutable. Blockchains use cryptography to encrypt information. Based on the unique accounting method of "Block+chain" (that is, blocks are stored in chronological chain), the information stored on the blockchain cannot be easily tampered with. (2) Traceability. Blockchain uses a unique and untamper-able distributed ledger to record informations of whole life cycle, informations cannot be modified or deleted but can be traced back chain by chain. (3) Decentralization. Unlike the traditional centralized system, attacks on one node will not lead to the entire network's collapse of blockchain. The completion of each transaction is a system of its own, the "precise" node data processing mode makes transactions more simple, convenient and higher efficiency, and less likely to cause massive information leakage. (4) Smart contracts. Which is programmed to be executed automatically once the terms are met. Because the corresponding conditions are retrieved by computer, there is no need to worry about denials.

\section{Exploration on the Application of Blockchain in Shared Employees}

\footnotetext{
* Corresponding Author
} 


\subsection{Create Trust Mechanism to Solve Technical Problems}

Blockchain technology irreversibly encrypts transaction information through hashing algorithms. That is, one cannot get plaintext of the original data through input information by working backward, which effectively prevent tampering. In hashing algorithms, the input data is different, and the output data is also random ones. In the 256-bit input data, any change will result in a different 32-bit output ones, which effectively ensures the security of on-chain data. While the data itself is always on the chain for query, so the integrity, stability and reliability of information can be guaranteed. The above consensus mechanism provides technical foundation for shared employees, and also builds a framework for information sharing (Fig. 1). The nodes on the chain can directly exchange information, resources and services they need. In addition, the blockchain system can also guarantee the security and reliability of information through time stamps, digital signatures and other technologies [3].

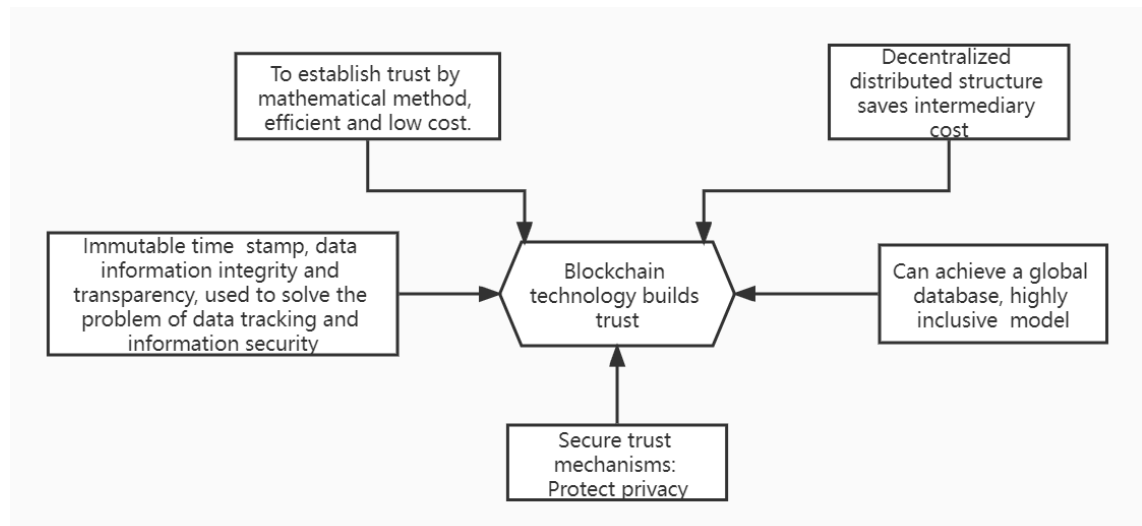

Fig. 1. Blockchain builds technical foundation for shared employees through trust mechanism

Under blockchain technology, trust is created by mathematical algorithms rather than marketing, advertising or online resources, which is reliable, low-cost and efficient. Informations about employees who want to join the platform can be stored on the blockchain, such as work history, insurance contributions, workers' compensation, job performance and evaluation, etc. Blockchain can turn such information into trusted, immutable content which can be stored or broadcast on the chain. In this way, no matter where the employee comes from, when the enterprise receive his application, all relevant and reliable information of this person under authorization can be read, then the enterprise can decide whether or not to hire the person immediately. In this way, many time or expenditure will be saved, for example, background checks, job trials and transactions with intermediaries, etc.

\subsection{Reducing Search Costs on Blockchain-based Credit Evaluation Systems}

Because of its flexible employment approach, "shared employees" model promoted across the country rapidly although it emerged in the context of THE COVID-19 pandemic. For example, the public Employment Association of West Coast New Area in Qingdao, Shandong, lenovo Wuhan Branch and so on. Even in 2021, when the epidemic has eased, the "shared employee" craze is still growing, and many new models have emerged. (1) Packet sharing mode - In the form of "shared", some cultural enterprises set up teams for cultural project planning, design, art, animation, publicity and marketing, to serve the government, enterprises and public institutions, etc. As a result, shared employees are no longer limited to low-end labor force, nor to just a few businesses. Instead, the demander of employment directly seeks suppliers through the platform [4-5]. (2) Express sharing mode - By socializing express delivery services, express delivery companies encourage idle members of the community and even on-the-job employees passing by after work to "share" express delivery services. In this way, anyone can be a courier [6].

Both of the above two models mean that "shared employees" have become social. This makes it necessary to establish a credit evaluation system both for employees and for enterprises involved in shared work. With the support of this system, whether an employee is suitable for "sharing" kind of work, can be determined almost instantly. The establishment of shared employees' credit evaluation system needs to be based on digital information system [7], and then the information of each volunteer to participate in the sharing plan will be digitized to be "on chain". Which includes his learning process, salary wanted, special skills, training and working experience, 
etc. Plants can publish its recruitment information such as salaries, working place in the form of label, then the enterprises can easily find matching employees through the credit evaluation system and the matched volunteer can easily find the enterprises, too. Based on the trustworthiness of the system, cooperation may be reached immediately. The system reduced the searching cost, and improved the two party's efficiency. Of course, the credit evaluation system be set up not only for employees but also for the enterprise. Based on the immutable nature of blockchain, some bad information, such as false recruitment or unpaid wages and other dishonest facts will be made known on chain forever, and the enterprise will certainly suffer the consequences.

\subsection{Blockchain Provides Assurance to Trace the Past}

Internet has memories, but what was left on it was often "anonymous", which make it difficult to prove where some information comes from. Blockchain looks like a "magic mirror", which makes bad information nowhere to hide. For example, traceability of employees' past work history and job evaluation will prevent him from acting recklessly. In short, blockchain will make it harder for unruliness guys exist and live happy.

\subsection{Blockchain can Facilitate Complex Shared Employee Projects}

Complex labor collaboration can be easily completed when blockchain is developed to a certain extent. For example, some large and complex integration projects, which requires Engineering technicians, information technology personnel, professional translators of some special languages and even overseas management personnel to complete cooperatively. Prior to the launch of blockchain technology, such collaboration was done through human resource intermediaries or through corporate bidding, which has many difficulties, e.g. time-consuming, difficult to check background, high intermediate fees. With the help of blockchain, enterprises need only to input project-related parameters in system to match labeled people. The system will automatically find suitable ones no matter from different industries, regions, or even from different countries. These people do not need to have a thorough understanding of the project, but are qualified and willing to work together to complete the assigned task at the assigned time.

\subsection{Smart Contracts Create Convenience for Shared Employees to Simplify Trading Process}

The trading process for smart contracts is shown as Fig. 2.

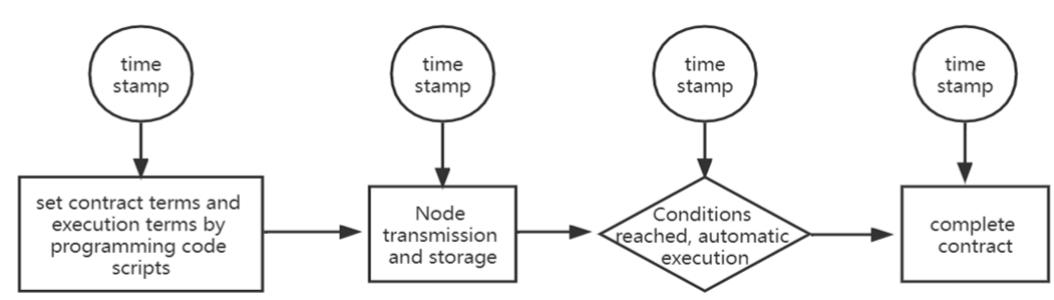

Fig. 2. Smart contract trading flow

The immutable nature of blockchain ensures the content of smart contracts. When a smart contract is executed, no one can modify the contract without trace, which can protect rights of both parties. Each node in the blockchain is a center of its own. When one node collapses, other nodes continue as usual, and its corresponding smart contract is as usual. Shared employees can take advantage of this property to achieve income distribution. Taking Hema Fresh as an example again, in addition to the basic salary, the shared employees also have "piece rate" income. When a customer placed an order, the information transmitted to employee A immediately, and employee A correctly completed his work, such as picking goods, weighing and bagging, which triggers the enforcement mechanism of smart contracts, once the customer confirms receipt, employee A's income from this order is credited to his account immediately according to the principle of smart contract [8]. 


\subsection{Blockchain Make Supervisions More Prudent and More Credible}

The immutability of blockchain can create trust, but blockchain itself is not everything. If an error occurs at the source of the upper chain, many errors will occur in the subsequent ones. This is where the authority entrusted to regulation can be exercised: when the supervisor discovers an error, they mark the correct ones on the chain, which will remain on the chain forever, if the supervisor mark an error message, the error ones will also remain forever, this makes regulation more prudent. To reduce or avoid remarks, the authority will strengthen the control of information source and information release, and strictly screen the on chain messages to ensure correctness. Over-reaching, freewheeling regulations can never appear again. For shared employees, employee information is no longer verified through identification, but through the private key. In the same way, one can't deny the traces he left on the chain. In summary, the way blockchain supports shared employees is summarized in Fig. 3.

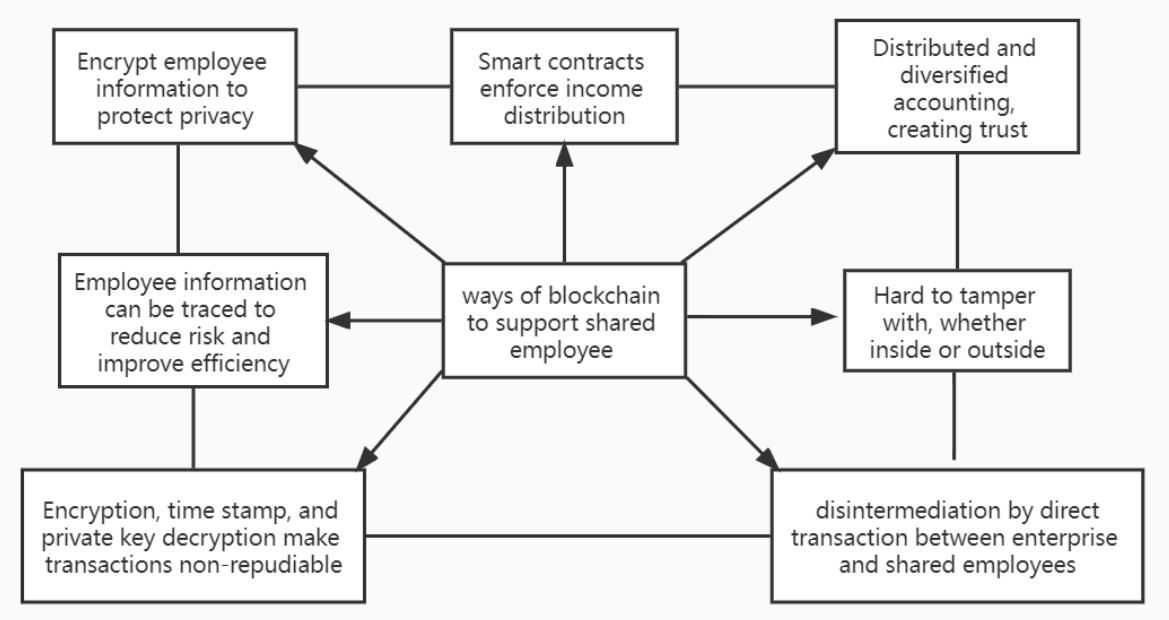

Fig. 3. Ways of blockchain to support shared employees

\section{Difficulties in Shared Employees Based on Blockchain Technology}

\subsection{Shared Employees are Mainly Labor-intensive and Simple Jobs Now}

Blockchain technology can record the workflow, work history, income and changes of simple tasks through distributed ledger, which is suitable for labor-intensive and low-end simple labor. For high-level labor, such as technology-intensive ones, or work that requires intellectual and creative participation, it is difficult to provide much help. For example, some designing work, even if shared employees have required work experience, it is difficult for him to "share" the job because of peer competition, trade secrets or other issues. The second difficulty in the "on chain" work is the aforementioned enterprise and individual's credit evaluation system, because many parameters in this system are difficult to define. For example, for team-type work, how to define the contribution rate of each person, especially between managers and technicians; another example, managers in the "management sharing" category are unable to define current productivity based on their historical contributions. Therefore, simple labor can easily participate in "sharing", while complex ones have many difficulties.

\subsection{The Evaluation Criteria of Workload are Inconsistent between Enterprises}

Shared employees can find jobs easily by blockchain. However, it is difficult for their new job to be completely consistent with their original ones, and the criteria of workload is inconsistent, either. So there is also inconsistency in the work performance evaluation. Take Hema Fresh as an example again, Hema Fresh pays the shared employees in the form of "fixed base plus piece rate" [9], he asked shared employees to complete a certain amount of work in a certain time, no additional performance evaluation given, so was the bonus. But in the shared employees' opinion, their work in Hema Fresh were not as easy as their original ones, their salary is lower not only than their original ones but also than Hema's own employees. Which doesn't mean sharing companies like Hema are bad, some difficulties still difficult to overcome especially for this kind of temporary job. In this case, blockchain "records" the workload of shared employees, but doesn't "work" on performance [10]. 


\subsection{Blockchain Offers only Technical Possibilities for Some Specific Jobs}

Some jobs cannot be "shared", such as employees with proprietary technology or those working on similar projects at competing companies, for legal, trade secrets, confidentiality agreements and other reasons. There are also jobs that only a few people qualified cannot be done via blockchain. Some other jobs that blockchain cannot help, for example, dangerous ones.

\subsection{The Establishment of Enterprise Evaluation System is Difficult}

Nominally, enterprises can evaluate employees, and employees can evaluate enterprises, especially enterprises with a large number of shared workers. For example, fresh stores, weekend and holiday business volume is significantly higher than usual, time requirements in summer are significantly higher than in winter. So it is difficult to establish a consistent evaluation system for enterprises on the blockchain, not only because the contents of operation are different between enterprises, but also working hours, work precision etc., even in the same enterprise, different seasons sometimes means different Job requirements. On the contrary, the establishment of employees' credit evaluation system will be easier, such as whether to keep faith, whether to complete the work with quality and quantity, whether there are customer complaints and so on.

\subsection{The Ability to Handle Large Transactions Needs to be Improved}

First of all, high energy consumption. Blockchain is based on mathematical encryption technology, so the operation requires a lot of calculation, the calculation process itself consumes a lot of energy. The more enterprises and employees join the blockchain, the more repetitive calculations, and the higher energy consumption. The demand for energy will obviously be greater if the whole transaction of people on chain put into operation. Secondly, high requirements for storage space. Blockchain stores and manipulates data through a specific independent, distributed database such as LevelIDB, as the volume of transactions increases, so does the amount of data stored and the amount of computation. When everyone's data is on chain simultaneity, data processing speed and system stability will be challenged.

\subsection{Blockchain Technology Still Has Room for Improvement}

First of all, there are challenges to consensus mechanism. In the future, as mathematical technology advances, some algorithms and the original encryption technology may be upgraded. Secondly, if the users' private key were stolen, some new security problems may arise. Thirdly, Blockchain matches information between supply and demand in the form of tags, but the authenticity and legality of the work cannot be promised in real life synchronously. The same transaction is legal and normal in some companies, may be criminal in other ones. The shared employees do the "normal" work on the basis of trust, and may not be able to define the legality of the work.so the problem of verifying authenticity may be faced.

\section{Conclusion and Prospect}

Shared employees is an inevitable result of urbanization to a certain extent. As a new mode of enterprise employment, "Shared employees" was created to cope with the short-term deployment of workers by enterprises, but in the long run. The fluctuation of labor demand will be the norm. If the Supply side and demand side can realize complementary through sharing platforms, the efficiency of social resource allocation will be improved naturally. With the development of science and technology, the universal blockchain maybe realized one day. At that time, everyone, whether employed or retired, may have the opportunity to participate in the "sharing" job in some form, and even go abroad to realize the global "sharing" employment. With the support of blockchain technology, it is possible to overcome many previously unimaginable problems, such as the connection of social security systems between countries. Maybe one day it is possible to work at home and "job-hopping" at home across the country and even around the world. In this way, everyone is not only an official employee of the enterprise in the country where he/she is located, but also can realize "shared employment" nationwide or even worldwide through. That day may come soon. 


\section{Acknowledgements}

The work is supported by the Science Developing Foundation of Beijing City University (Grant KYFZ202001): Feasibility Study on Shared Employees Based on Blockchain Technology.

\section{References}

[1] D.T. Zhuang, Can "shared employees" become a new mode of sharing enocomy, Network of Democracy and Law, 202002-09.

[2] Technical specification for financial distributed ledger security (JR/T 0184-2020), People's Bank of China, 2020-02-25.

[3] L. Zhang, B.X. Liu, R.Y. Zhang, A Review of Blockchain Technology, Computer Engineering (2019) 1-15.

[4] W.J. Ge, A new mode of knowledge management package sharing--From the perspective of sharing economy, Special zone economy (08)(2021) 67-74.

[5] X.D. Cheng, F. Zhang, P.M.A. Baker, The reconstruction and promotion of labor by gig economy and sharing economy, Chinese soft science (1)(2020) 21-29.

[6] Z.Q. Lin, The new trend of express delivery industry under "sharing mode", Logistics technology and application (24)(10) (2019) 96-99.

[7] X.R. Gao, Y. Shi, Research on Credit Constraint Mechanism of Sharing Economy Based on Blockchain Technology, Credit Reporting (7)(2020) 26-32.

[8] R.H. Yang, Application of Blockchain Technology in Financial Sharing Field, The Accounting Issue (9)(2020) 35- 40.

[9] X. Wang, H.Q. Liu, Discussion on Employment Mode of Retail Enterprises under the Background of Sharing Economy-A Case Study of Hema Fresh, Business Economics Research (5)(2021) 136-138.

[10] P.Y. Xiao, Sharing by Law: A Study On The Legal Risk of Sharing Employees and Its Prevention and Control, China Human Resource Development (7)(2020) 96-106. 